\title{
Frequency characteristics of a GPL-reinforced composite microdisk coupled with a piezoelectric layer
}

\begin{abstract}
This is the first research on the frequency analysis of a graphene nanoplatelet composite (GPLRC) microdisk in the framework of a numerical-based generalized differential quadrature method. The stresses and strains are obtained using the higher-order shear deformable theory. Rule of mixture is employed to obtain varying mass density, thermal expansion, and Poisson's ratio, while module of elasticity is computed by modified Halpin-Tsai model. Governing equations and boundary conditions of the GPLRC microdisk covered with piezoelectric layer are obtained by implementing Hamilton's principle. Regarding perfect bonding between the piezoelectric layer and core, the compatibility conditions are derived. In addition, due to the existence of piezoelectric layer, Maxwell's equation is derived. The results show that outer-to-inner ratio of radius (Ro/Ri), ratios of length scale and nonlocal to thickness $(1 / \mathrm{h}$ and $\mu / \mathrm{h})$, ratio of piezoelectric to core thickness $(\mathrm{hp} / \mathrm{h})$, applied voltage, and GPL weight fraction (gGPL) have significant influence on the frequency characteristics of the GPLRC microdisk. Another important consequence is that in addition to the nonlinear indirect effects of applied voltage on the natural frequency of the GPLRC microdisk covered with piezoelectric for each specific value of $\mathrm{Ro} / \mathrm{Ri}$, the impact of the $\mathrm{Ro} / \mathrm{Ri}$ on the natural frequency is indirect. A useful suggestion of this research is that, for designing the GPLRC circular microplate at the low value of the Ro/Ri should be more attention to the gGPL and Ro/Ri, simultaneously.
\end{abstract}

